- Europhysics News: reduction in the production costs by supplying material in electronic form to the printer and increased advertising income should net $25 \mathrm{kSFR}$ each year;

- Members: a continuing steady increase in the number of IOM's (100 per year equivalent to $5 \mathrm{kSFR}$ seems reasonable);

- Associate Members: encouraging existing members to increase their support and finding new Associates should yield an additional income of 20 kSFR. The Treasurer notes that the response to initiatives by a few individuals has met with an excellent initial response. Five organizations have recently agreed to become Associates and four others will raise their contributions (giving a total of about 20 kSFR).

\section{Scenarios}

All in all, an additional $70 \mathrm{kSFR}$ of income above the current level seems achievable. But is it enough? Unfortunately not. One problem is inflation that appears to be peaking at $6.5 \%$ in Switzerland where most of the Society's expenses are incurred. G. Thomas has made a detailed analysis of various scenarios for the evolution of the Society's finances. It shows that in order for him to stay just two years in Budapest (until the beginning of 1993), and assuming an inflation rate of $5 \%$, then the deficit will only be reduced to zero if income is increased by about 150 kSFR from today's amount. So this is clearly the long-term requirement: we have to raise an additional 80 kSFR, above the 70 kSFR that can be expected, to ensure that Gero Thomas returns to Geneva and we have a no deficit in 1993 and beyond.

Sources of this additional income are limited. One is clearly us Members as it is essentially we who must meet the Society's responsibilities. The Treasurer aims to submit two proposals to Council in March and Members had until 6 January to comment. The first calls for an increase in the unit fee from 12 to 13.5 SFR on 1 January 1992 corresponding to an annual increase of just under $4.5 \%$ since the last revision that came into effect in 1989. If agreed it would raise income by $60 \mathrm{kSFR}$, thus meeting $75 \%$ of the requirement. The second is indexation of the unit fee to the Swiss rate of inflation: its implementation would raise about 15 kSFR in 1993.

The overall result of the two proposals still leaves us a little short of the target of the equivalent in 1991 of 80 kSFR in new income. Considerable progress in tackling the problem has been achieved and a concerted effort is underway to further expand fund raising initiatives involving donations and the Associates. The President is also studying the problem of non-convertible currency with a view to making some concrete proposals. IOM's, the Divisions and the national societies are meanwhile being urged to carry out membership campaigns as a matter of urgency to ensure the sound financing that will allow the Society's activities to continue to be improved.

\title{
The Dynamic Sun
}

Somewhat appropriately, the sixth triennial meeting in Debrecen, Hungary last May of the Solar Physics Section of EPS just about corresponded with a maximum in a cycle of solar activity. It was also the first time the conference was held in an east European country, where flawless organization by the local organizers (L. Dezsö and coworkers) in the face of a more difficult economic situation than on previous occasions was greatly admired and appreciated. Equally remarkable was the appearance by October of the proceedings as a Publication (Vol. 7) of the Debrecen Heliophysical Observatory, Hungary.

The label "The Dynamic Sun" was chosen to cater for a large fraction of the solar community and its broad range of interests covering the structure and dynamics of the solar atmosphere. Selected aspects such as the solar wind and processes in the Sun's

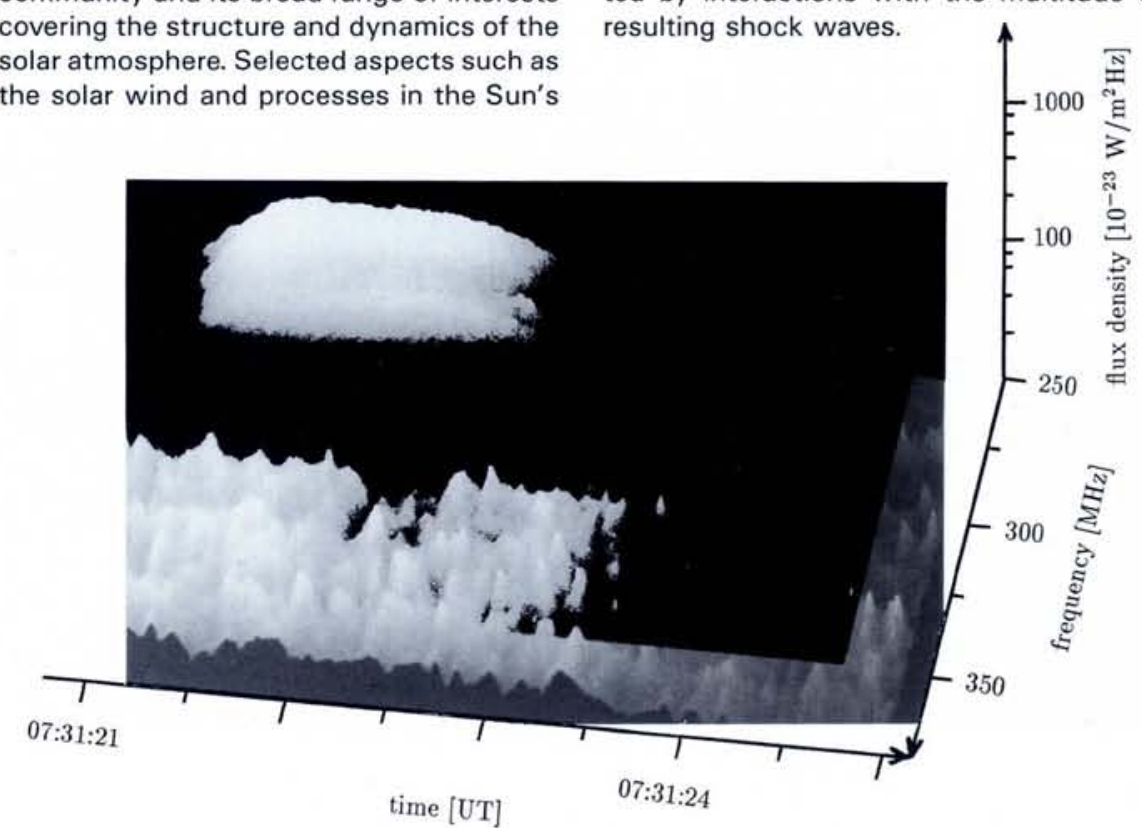

Fig. 1 - Fragmentation of a solar flare on the finest scale as shown by a radio spectrum made at the ETH Zürich (Benz, 1990). The flux density is plotted in three dimensions as a function of time and the frequency, where the observing frequency represents the plasma frequency in the source and is proportional to the square root of the particle density. The spikes of flux in the lower part of the spectrogram, which originate at the sites of energy release in the regions of high density at low altitudes, suggest that the release is extremely fragmented. The more gentle release shown in the upper part is for emission from the low density region at altitudes which are some $50000 \mathrm{~km}$ higher: it is the signature of an escaping beam of energetic electrons traversing the corona.

interior were excluded as they had been adequately dealt with at recent meetings.

\section{Large-scale motions from the interior}

Motions on scales exceeding the supergranules, the largest structures of the quiet photosphere, were reviewed by $\mathrm{H}$. Wöhl (Kiepenheuer-Institut) and I. Tuominen (Helsinki). It has become clear that solar activity is connected with anomalies of solar rotation rather than with homogeneous differential rotation. Evidence in favour of an almost rigid rotation of the strongest magnetic fields and of complexes of activity was presented. The weak, large-scale background, however, rotates with rates depending on the latitude. Its newly found vorticity patterns also received attention.

Release of magnetic energy leads to particle acceleration

The release of magnetic energy is conocticle accelation and, in turn, lengths. G. Trottet (Meudon Observatory) and L. Vlahos (Thessaloniki) reviewed the observations of flare-initiated emissions. They can now be divided into five phases of energy build-up, release and transformation. A most relevant recent discovery, based largely on radio observations, is that posed of thousands of microflares (Fig. 1). Theoretical modelling assumes a global instability breaking up into fast local explosions: particles can then be easily accelerated by interactions with the multitude of resulting shock waves.

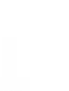


This scenario requires small transverse scales in the magnetic field and even if the photospheric motions are slow, there is generally no equilibrium state. The braiding of field lines produces discontinuous field variations in a large number of small regions
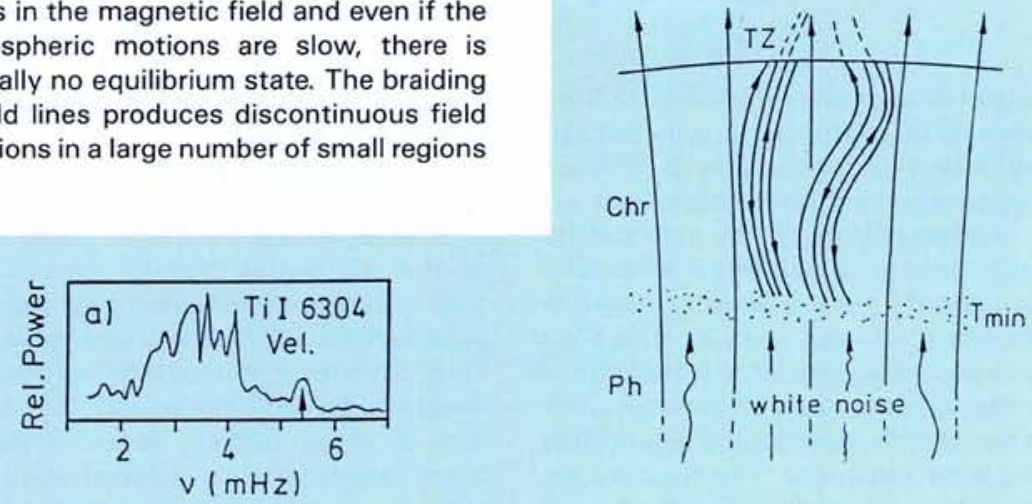

Fig. 2 - Chromospheric oscillations: (a - left) The power spectrum of velocity oscillations in the dark regions of a sunspot umbra measured using the Doppler shift of the $\mathrm{Ti}$ I spectral line at $6304 \AA$. The broad oscillation centered on $3 \mathrm{mHz}$ originates in the photosphere and the much weaker (arrowed) oscillation at $5.5 \mathrm{mHz}$ in the chromosphere (Abdelatif et al., 1986).

(b - right) Chromosphere resonator model (Zugzda at al., 1984) where the sunspot comprises a vertical arrangement of more or less coupled resonating cavities in which the various modes of magneto-atmospheric waves are partially trapped and partially transmitted. Waves from the photosphere (Ph) enter the chromosphere (Chr) resonator defined by lower and upper boundaries (at the minimum temperature $T_{\min }$ and the transition zone $T Z$ into the solar corona). Multiple reflections give high transmission at specific wavelengths so modelling the spectrum of resonance frequencies (a) can provide, for example, a seismictype determination of the distance between $T_{\min }$ and $T Z$.

Fig. 3 - Temperature variations in solar flares. The observed variation with time of the ratio of the temperatures of $\mathrm{Fe}$ and $\mathrm{Ca}$ ions in a flare measured during the Solar Maximum Mission using the intensities of the $J$ and $W$ spectral lines of $F e$ and the $K$ and $W$ lines of $\mathrm{Ca}$. The ratio increases during the period of maximum flare heating (between the vertical dashed lines) accompanying the thermalization of electromagnetic energy. Modelling indicates that the temperature across the top of the flare is non-uniform and that the flare has a relatively small, hot core (Sylwester, 1990).

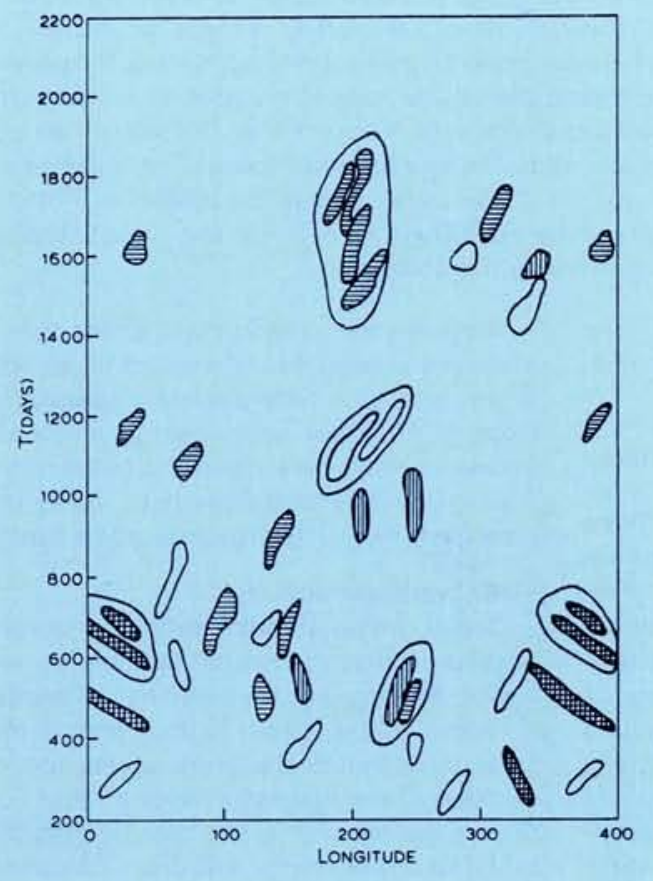

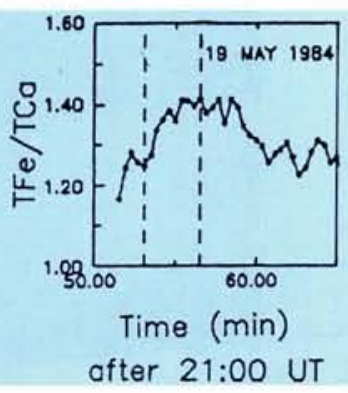

Fig. 4 - A longitude-time diagram showing composite nests of sunspot activity (circled) for four narrow ranges of latitude in the northern hemisphere of the Sun's surface (Zwaan, 1990). Nests with more than four sunspot groups selected by a particular clustering criterion are plotted for the latitude ranges $<10^{\circ}$ (blank), $10-15^{\circ}$ (horizontally hatched), 15-200 (vertically hatched), 20-30 (cross-hatched).

The presence of composite nests holds clues to the Sun's magnetic structure and the basic mechanism of solar activity. One idea is that the same bundle of toroidal magnetic flux may have several loops (like the Loch Ness monster). An active sunspot regions unfolds as each of the loops rises and emerges into the atmosphere. This could explain why the nests making up a composite nest overlap in time, and why their latitudes and longitudes differ by small amounts. and it is these elementary hot spots that ultimately heat the corona.

\section{Waves in active regions}

Waves and oscillations in the solar atmosphere have attracted the attention of astronomers for many years as they are candidates for energy transfer and heating in stellar atmospheres. Magneto-atmospheric waves in active regions must be considered, i.e. three restoring forces - pressure (compressibility), gravity (buoyancy) and the magnetic field - operate. Recent advances in observational techniques (the vacuum solar telescopes at Tenerife, CCD cameras) permit the resolution of many details of these periodic disturbances (Fig. 2a) and of the basic atmospheric structure (Fig. 2b).

Observations and updated models of both waves and atmospheres in active regions were reviewed by F. Kneer (Göttingen) and Z.D. Zugzda (IZMIRAN, Moscow). The predicted oscillations depend critically on the assumed mean structure of the atmosphere, the convective zone and the magnetic field. Comparing predictions with observations yields a useful method for probing the atmosphere's structure. Seismic techniques, which have been applied with great success to the study of the Sun's interior, can now also be used for sounding the more complex structures in the active regions of the solar atmosphere.

\section{Corona - the Sun's upper atmosphere}

The solar corona, a plasma at $10^{6} \mathrm{~K}$ and the realm of UV, EUV and radio emissions, was reviewed by $\mathrm{H}$. Mason (Cambridge) and J. Sylwester (Wroclaw). Up-to-date understanding of line emission processes has provided new insights into density and temperature distributions in active regions and flares (Fig. 3). Space missions to be launched shortly, such as Solar A, Coronas and $\mathrm{SOHO}$, give rise to the expectation of high resolution spectral data for examining the upper atmosphere of the Sun and, in particular, the origin of its high temperature.

Patterns of activity and the solar dynamo

It is well known that the main belts of solar activity cover the Sun's equatorial region at up to $40^{\circ}$ of heliographic latitude. The pattern of activity has been investigated for several decades and until recently the concept of active longitudes was widely adopted, with activity occurring repeatedly in the same longitudes, but at changing latitudes. C. Zwaan (Utrecht) demonstrated in his review talk that instead of active longitudes the new approach of activity nests should be favoured. These patterns of activity contain more sunspots than expected by chance and they have rather large extensions in latitude and longitude. The nest structure indicates the presence of fields on the order of $10^{4}$ gauss in the dynamo region near the bottom of the convection zone. There is also an intriging tendency for the nests to cluster once again into nests of nests (called composite nests - Fig. 4).

A.O. Benz ETH Zürich 